\title{
Outcomes in conventional laparoscopic versus robotic-assisted primary bariatric surgery: a retrospective, case-controlled study of the MBSAQIP database
}

\author{
Edwin Acevedo Jr. ${ }^{1} \cdot$ Michael Mazzei $^{1} \cdot$ Huaqing $\mathrm{Zhao}^{2} \cdot$ Xiaoning Lu $^{2} \cdot$ Rohit Soans $^{1} \cdot$ Michael A. Edwards $^{3}$
}

Received: 20 December 2018 / Accepted: 12 June 2019 / Published online: 17 June 2019

(c) Springer Science+Business Media, LLC, part of Springer Nature 2019

\begin{abstract}
Introduction Robotic-assisted bariatric surgery is increasingly performed. There remains controversy about the overall benefit of robotic-assisted (RBS) compared to conventional laparoscopic (LBS) bariatric surgery. In this study, we used a large national risk-stratified bariatric clinical database to compare outcomes between robotic and laparoscopic gastric bypass (RNYGB) and sleeve gastrectomy (SG).

Methods A retrospective analysis of the 2015 and 2016 Metabolic and Bariatric Surgery Accreditation and Quality Improvement Program (MBSAQIP) Participant Use Data File (PUF) was performed. Primary robotic and laparoscopic RYNGB and SG were analyzed. Descriptive analysis was performed of the unmatched cohorts, followed by 1:3 case-controlled matching. Cases and controls were matched by patient demographics and pre-operative comorbidities, and peri-operative outcomes compared.

Results 77,991 Roux-en-Y gastric bypass (RnYGB) (7.5\% robotic-assisted) and 189,503 SG (6.8\% robotic-assisted) cases were identified. Operative length was significantly higher in both the robotic-assisted RnYGB and SG cohorts $(p<0.0001)$. Outcomes were similar between the robotic-assisted and laparoscopic RnYGB cohorts, except a lower mortality rate $(p=0.05)$, transfusion requirement $(p=0.005)$, aggregate bleeding $(p=0.04)$, and surgical site infections (SSI) $(p=0.006)$ in the robotic-assisted cohort. Outcomes were also similar between robotic-assisted and laparoscopic SG, except for a longer length of stay $(p<0.0001)$ and higher rates of conversion $(p<0.0001)$, 30-day intervention $(p=0.01)$, operative drain present $(p<0.0001)$, sepsis $(p=0.01)$, and organ space SSI $(p=0.0002)$ in the robotic cohort. Bleeding was lower in the robotic SG cohort and mortality was similar.

Conclusion Both robotic-assisted and laparoscopic RnYGB and SG are overall very safe. Robotic-assisted gastric bypass is associated with a lower mortality and morbidity; however, a clear benefit for robotic-assisted SG compared to laparoscopic SG was not seen. Given the longer operative and hospital duration, robotic SG is not cost-effective.
\end{abstract}

Keywords Primary bariatric surgery $\cdot$ Robotic-assisted $\cdot$ Conventional laparoscopic

Electronic supplementary material The online version of this article (https://doi.org/10.1007/s00464-019-06915-7) contains supplementary material, which is available to authorized users.

Michael A. Edwards

edwards.michael@mayo.edu

1 Department of Surgery, Division of Bariatric and Minimally Invasive Surgery, Temple University Hospital, Philadelphia, PA, USA

2 Department of Clinical Sciences, Lewis Katz School of Medicine at Temple University, Philadelphia, PA, USA

3 Department of General Surgery, Mayo Clinic, 4500 San Pablo Rd. S, Jacksonville, FL 32224, USA
Obesity is a worldwide epidemic. According to the World Health Organization (WHO), in 2016 more than 1.9 billion adults were overweight and over 650 million were classified as obese globally [1]. Obesity is a preventable disease that is caused by excessive caloric intake, fat accumulation, and fat dysregulation, and is associated with considerable deleterious downstream effects. It has been found to significantly increase the risk of chronic diseases, such as obstructive sleep apnea, diabetes, cardiovascular disease, hypertension, and even some cancers [2]. In 1967, Edward E. Mason introduced the concept of surgical gastric restriction for the treatment of morbid obesity [3]. Since then, techniques in bariatric surgery have been refined and many studies report 
successes in sustaining weight loss and resolution of, or improvement in comorbid conditions [4-8]. In fact, in 2014 the National Institutes of Health (NIH) held a consensus panel to review evidence regarding its safety and efficacy in treating obesity. They concluded that there is sufficient evidence to support metabolic and bariatric surgery for the treatment of obesity and type 2 diabetes mellitus (T2DM) [8].

Surgical approaches to weight loss continue to evolve. Technologies such as laparoscopic and robotic-assisted surgical systems have revolutionized metabolic and bariatric surgery to the point where many of these operations can be performed in an outpatient setting, or require a relatively short hospital length of stay (HLOS). Many studies have compared laparoscopic (LBS) and robotic-assisted (RBS) metabolic and bariatric surgery with varied results [4, 9-20]. Baily et al. performed a systematic review and economic analysis that compared laparoscopic and robotic-assisted Roux-en-Y gastric bypasses, and found that complication rates, operative outcomes, and HLOS did not differ significantly between the two surgical approaches [9]. In a more recent systematic review and meta-analysis, $\mathrm{Li}$ et al. found that there were no significant differences between conventional laparoscopic (LBS) and robotic-assisted (RBS) metabolic and bariatric surgery in overall post-operative complications, major complications, readmission, conversion, or mortality [10]. They also noted that robotic-assisted cases were associated with longer operative time and HLOS when compared to laparoscopic procedures, in contrast to findings by Bailey et al. [9]. Although this most recent systematic review and meta-analysis included 34 observational studies, only 27,997 patients were included in the meta-analysis. In fact, many of the studies evaluating laparoscopic and robotic-assisted bariatric surgery have been limited by small numbers and have reported mix results. Here, we present one of the largest retrospective case-control studies of the Metabolic and Bariatric Surgery Accreditation and Quality Improvement Program (MBSAQIP) Participant User Files (PUF) database comparing peri-operative outcomes between conventional laparoscopic and robotic-assisted primary Roux-en-Y gastric bypass (RnYGB) and sleeve gastrectomy (SG) cases.

\section{Materials and methods}

\section{Data source and case selection}

We performed a retrospective analysis of the 2015 and 2016 Metabolic and Bariatric Surgery Accreditation and Quality Improvement Program Participant Use File (MBSAQIP PUF) for this study comparing primary robotic-assisted and conventional laparoscopic metabolic and bariatric surgery. The MBSAQIP PUF is the largest bariatric-specific clinical database. It collects prospective risk-adjusted data based on standardized definitions. Data are collected by trained clinical nurse reviewers at each bariatric center and audited similar to the National Surgical Quality Improvement Program (NSQIP). De-identified data are reported on patient characteristics, operative details, and intra-operative and peri-operative outcomes. An Institutional Review Board (IRB) approval was not required as the study utilized only de-identified data from a nationally available clinical database.

There are 355,675 bariatric cases in combined 2015 and 2016 MBSAQIP PUF. We selected primary gastric bypass and sleeve gastrectomy cases by Current Procedural Terminology (CPT) codes 43644, 43645, and 43775. Inclusion and exclusion criteria are detailed in Fig. 1. Cases stratified by revision/conversion variable were first excluded. Open metabolic and bariatric procedures (CPT codes 43845, 43663, 43846, and 43847) were also excluded. Cases were then selected by variable "Surgical_Approach," limiting selection to only conventional laparoscopic and robotic-assisted cases. Cases with missing data were additionally excluded from analysis.

\section{Case-control matching}

In order to control for variables that may influence outcomes comparing the conventional laparoscopic and robotic-assisted cohorts, cases and controls were matched by patient demographics (age, gender, race/ethnicity, and body mass index (BMI) closest to surgery), functional status (ASA classification, limited ambulation status, partial functional dependence, and total functional dependence), and pre-existing comorbid conditions (hypertension requiring medication, hyperlipidemia, myocardial infarction, renal insufficiency, chronic renal disease requiring dialysis, vein thrombosis requiring therapy, history of pulmonary emboli, diabetes mellitus, current smoking within 1-year, obstructive sleep apnea, chronic obstructive lung disease, oxygen dependence, and steroid/immunosuppressant use). An initial 1:3 case-control matching was performed with the above covariates for both sleeve gastrectomy and gastric bypass cases independently. In order to account for surgeon experience as a possible confounder, a second procedure-specific 1:3 case-control match analysis was performed, controlling for operative duration and conversion, as these variables may correlate with surgeon experience-a variable that is missing from the MBSAQIP PUF database. In addition to the patient demographics and pre-operative comorbidities included in the first matched analysis, operative duration and conversion rate were included as matching covariates in the second procedure-specific case-control match analysis. 


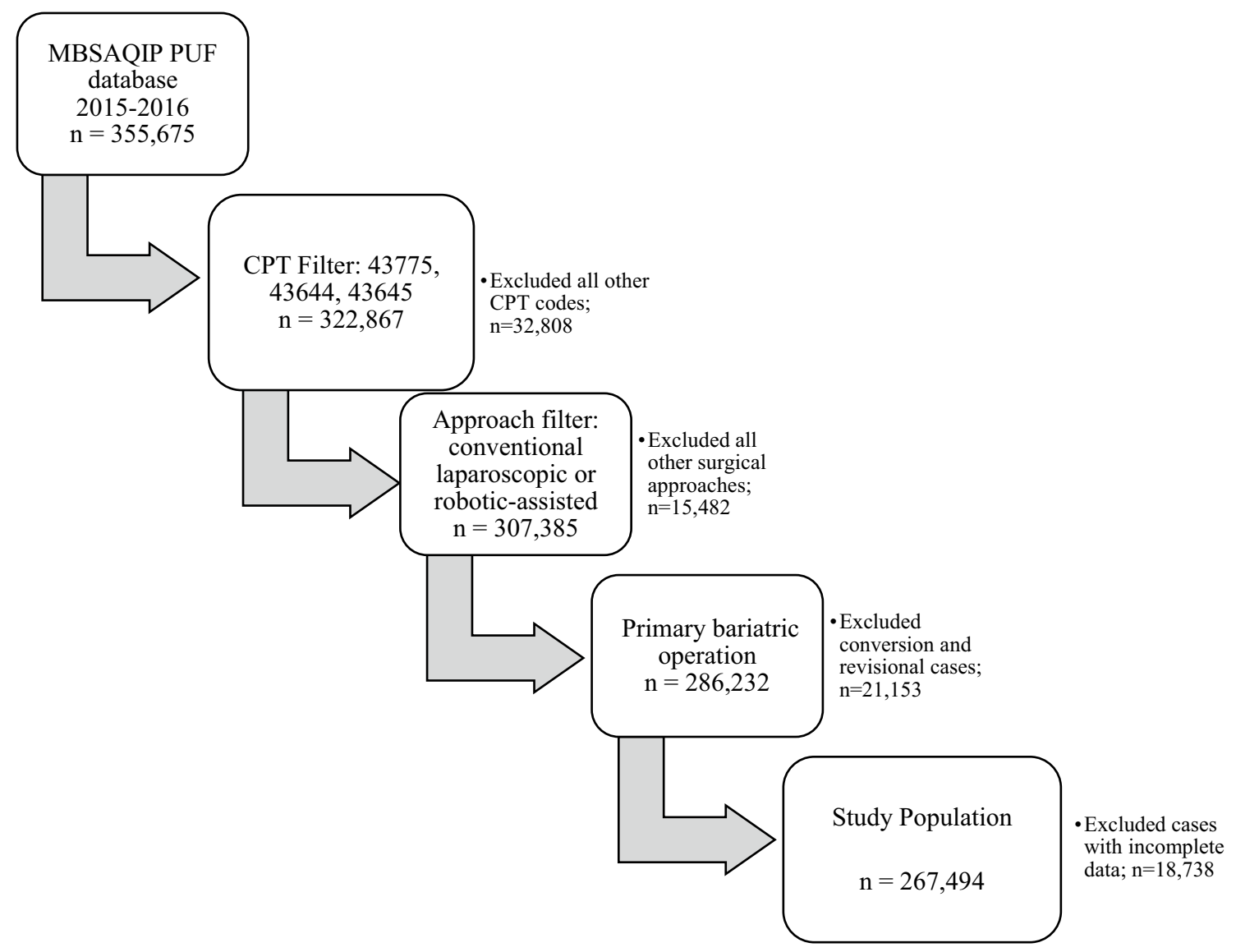

Fig. 1 Case selection flow diagram. Flow diagram outlining case inclusion and exclusion criteria. MBSAQIP PUF Metabolic and Bariatric Surgery Accreditation and Quality Improvement Project Participant Use Data File, CPT CPT

\section{Outcome measures}

Thirty-one primary outcome variables were assessed, including operative length, HLOS, conversion rate, discharge status, 30-day ICU admission, reoperation, readmission, intervention or mortality, drain present at 30-days, death likely related to bariatric surgery, renal failure, progressive renal insufficiency, cardiopulmonary resuscitation (CPR), coma $>24 \mathrm{~h}$, stroke, myocardial infarction (MI), venous thrombosis requiring therapy, pulmonary emboli (PE), anticoagulation for presumed/confirmed vein thrombosis/PE, transfusion, pneumonia, unplanned intubation, on ventilator $>48 \mathrm{~h}$, urinary tract infection (UTI), sepsis, septic shock, superficial surgical site infection (SSI), deep SSI, and organ space SSI. Seven aggregate complications were also assessed, including aggregate leak, bleeding, renal, cardiovascular and pulmonary complications, venous thromboembolic events, and surgical site infections. These composite complications methodology has been previously reported by Berger et al. [21] and are based on variables in the Related File of the MBSAQIP PUF. For example, aggregate leak is defined by 30-day reoperation, readmission or intervention with the suspected reason being a "leak", the presence of a drain at 30-days, and organ space SSI. Outcome measures were analyzed and reported for gastric bypass and sleeve gastrectomy unmatched and matched patient cohorts.

\section{Statistical analysis}

Univariate analysis of patient demographics and comorbid conditions was performed using Pearson's Chi squared test for categorical variables (gender, race, ASA class, preoperative comorbidities) and an independent sample $t$ test and Mann-Whitney test for normally and non-normally distributed continuous variables (age, weight, and BMI), respectively. Univariate analysis of primary and aggregate outcomes was also performed using Pearson's Chi squared test for categorical variables (conversion rate, 30-day outcomes, and aggregate complication rates) and an independent sample $t$ test and Mann-Whitney test for normally and skewed distributed continuous variables (operative length, HLOS), respectively. Categorical variables were reported as frequency and percentage, and continuous variables as mean ( \pm standard deviation). A similar statistical analysis 
was performed for all matched cohorts. All statistical analysis was performed with SPSS version 25 (IBM Corporation, Armonk, NY) or SAS version 9.4 (SAS Institute, Cary, NC). A $p$-value $<0.05$ was considered statistically significant.

\section{Results}

Of the 355,675 cases in the 2015 and 2016 MBSAQIP PUF database, 77,991 Roux-en-Y gastric bypass (RnYGB) (7.5\% robotic-assisted) and 189,503 sleeve gastrectomy (SG) (6.8\% robotic-assisted) cases were identified. Descriptive statistics for unmatched RnYGB and SG cases are detailed in Table 1. For the entire unmatched RnYGB cohort, the mean age and BMI were 45.3 years and $46.3 \mathrm{~kg} / \mathrm{m}^{2}$, respectively. The unmatched robotic-assisted RnYGB cohort was older (46.5 years vs. 45.2 years, $p<0.0001$ ) with a lower BMI $\left(46 \mathrm{~kg} / \mathrm{m}^{2}\right.$ vs $\left.46.4 \mathrm{~kg} / \mathrm{m}^{2}, p=0.007\right)$. The robotic-assisted RnYGB cohort had a higher prevalence of numerous preoperative comorbid conditions, including myocardial infarction, hypertension, hyperlipidemia, renal insufficiency, impaired ambulation, obstructive sleep apnea (OSA), and chronic obstructive lung disease (COPD). The mean age and BMI were 44.4 years and $45.2 \mathrm{~kg} / \mathrm{m}^{2}$ for all sleeve gastrectomy cases. The unmatched robotic-assisted SG cohort had a higher BMI $\left(45.5 \mathrm{~kg} / \mathrm{m}^{2}\right.$ vs. $\left.45.2 \mathrm{~kg} / \mathrm{m}^{2}, p<0.0001\right)$, but were similar in age. This cohort also had a lower proportion of males ( $20 \%$ vs. $21.1 \%, p=0.002)$ and a higher proportion of Non-Hispanic Black patients $(20.1 \%$ vs. $18.3 \%$, $p<0.0001)$. Pre-operative comorbid conditions were mostly similar between the robotic-assisted and conventional laparoscopic sleeve gastrectomy cohorts.

30-day outcomes and peri-operative complications in the unmatched RnYGB and SG cohorts are outlined in Table 2. For both RnYGB and SG, operative length and conversion rate were significantly higher in the robotic-assisted cohort. Post-operative length of stay was significantly higher in the robotic-assisted sleeve gastrectomy cohort (1.8 days vs. 1.6 days, $p<0.0001$ ), but were similar between the robotic and laparoscopic gastric bypass cohorts $(p=1.0)$. For gastric bypass, all outcome measures were lower in the roboticassisted cohort, except for a higher rate of readmission (6.6\% vs. $5.6 \%, p=0.004)$. The rate of transfusion, superficial SSI, and aggregate wound infection were higher in the conventional laparoscopic cohort. All other outcome measures were similar between the gastric bypass cohorts. In contrast, 30 -day readmission $(3.6 \%$ vs. $3.2 \%, p=0.02)$, intervention $1.3 \%$ vs. $0.09 \%, p=0.0002$ and the present of an operative drain $(0.5 \%$ vs. $0.2 \%, p>0.0001)$ were significantly higher in the robotic-assisted sleeve gastrectomy cohort. Ventilator requirement $>48 \mathrm{~h}(0.1 \%$ vs. $0.05 \%, p=0.0008)$, sepsis $(0.1 \%$ vs. $0.08 \%, p=0.02)$, organ space SSI $(0.3 \%$ vs $0.2 \%, p<0.0001)$, aggregate leak ( $0.4 \%$ vs. $0.2 \%, p=0.003)$, and aggregate pulmonary complications $(0.5 \%$ vs. $0.3 \%$, $p=0.04$ ) were also higher in this cohort of patients. All other outcome measures were similar between the robotic and laparoscopic SG cohorts.

Roux-en-Y gastric bypass and sleeve gastrectomy robotic-assisted and conventional laparoscopic cases were then matched on demographics and pre-operative comorbid conditions. Descriptive statistics of the 1:3 matched cohorts are detailed in Table 3, including 12,688 Roux-en-Y gastric bypass (3172 robotic-assisted and 9516 conventional laparoscopic) and 35,956 sleeve gastrectomy (8989 roboticassisted and 26,967 conventional laparoscopic) cases. Compared to RnYGB, there was a higher prevalence of male (16.2\% vs. $14.3 \%)$, Black (18.6\% vs. $12.5 \%$ ), and Hispanic (9.6\% vs. $7.2 \%$ ) patients in the SG cohort. Demographics (age, gender, pre-operative weight, and BMI), ASA and pre-operative comorbidities were similar between matched robotic-assisted and conventional laparoscopic cohorts, for both Roux-en-Y gastric bypass and sleeve gastrectomy cases.

Outcomes for matched robotic-assisted and conventional laparoscopic Roux-en-Y gastric bypass and sleeve gastrectomy cases are reported in Table 4. For RnYGB cases, operative length (151.9 min vs. $114.6 \mathrm{~min}, p<0.0001$ ) and 30-day readmission $(6.6 \%$ vs. $5.6 \%, p=0.03)$ were significantly higher in the robotic-assisted cohort, while transfusion (1.0 vs. $0.5 \%, p=0.0005)$, aggregate bleeding $(0.8 \%$ vs. $0.4 \%$, $p=0.03)$, and aggregate wound infection ( $1.5 \%$ vs. $0.8 \%$, $p=0.006$ ) were significantly higher in the conventional laparoscopic cohort. There was no mortality difference $(0.1 \%$ vs. $0 \%, p=0.05)$. Post-operative length of stay and all other 30-day, non-aggregate and aggregate complications were similar between the robotic-assisted and conventional laparoscopic gastric bypass cohorts. For sleeve gastrectomy cases, operative length (99.2 min vs. $71.6 \mathrm{~min}, p<0.0001$ ), post-operative length of stay (1.7 days vs. 1.6 days, $p<0.0001)$, conversion rate $(0.6 \%$ vs $0.04 \%, p<0.0001)$, 30 -day intervention $(1.1 \%$ vs $0.8 \%, p=0.009)$, operative drain present at 30 -days $(0.6 \%$ vs $0.1 \%, p<0.0001)$, sepsis $(0.1 \%$ vs $0.05 \%, p=0.01)$, and organ space SSI $(0.3 \%$ vs $0.1 \%, p=0.0002$ ) were significantly higher in the roboticassisted cohort. Acute renal failure $(0.09$ vs $0.02 \%, p=0.03$ ) was significantly higher in the conventional laparoscopic cohort. All other outcome measures were statistically similar between the robotic-assisted and conventional laparoscopic sleeve cohorts.

The second 1:3 case-control match analysis was performed, which included operative length and conversion rate (surrogates for surgeon experience) as matching covariates. 7576 Roux-en-Y gastric bypass (1894 robotic-assisted and 5682 conventional laparoscopic) and 25,164 sleeve gastrectomy (6291 robotic-assisted and 18,873 conventional laparoscopic) cases were identified. Outcomes were compared and are detailed in Table 5. For Roux-en-Y gastric 
Table 1 Descriptive statistics in unmatched Roux-en-Y gastric bypass and sleeve gastrectomy cohorts

\begin{tabular}{|c|c|c|c|c|c|c|c|c|}
\hline & \multicolumn{4}{|c|}{ Roux-en- Y gastric bypass } & \multicolumn{4}{|c|}{ Sleeve gastrectomy } \\
\hline & All $[\mathrm{n}=77,991]$ & RGB $[\mathrm{n}=5817]$ & $\begin{array}{l}\text { LGB } \\
{[n=72,174]}\end{array}$ & P-value & $\begin{array}{l}\text { All } \\
{[n=189,503]}\end{array}$ & $\begin{array}{l}\text { RSG } \\
{[n=12,912]}\end{array}$ & $\begin{array}{l}\text { LSG } \\
{[n=176,591]}\end{array}$ & $p$-value \\
\hline \multicolumn{9}{|c|}{ Continuous variables, mean \pm SD } \\
\hline Age (years) & $45.3 \pm 11.9$ & $46.5 \pm 12.0$ & $45.2 \pm 1.9$ & $<0.0001$ & $44.4 \pm 12.0$ & $44.5 \pm 12.1$ & $44.35 \pm 12.0$ & 0.4 \\
\hline $\begin{array}{r}\text { Pre-operative } \\
\text { weight (lbs) }\end{array}$ & $284.5 \pm 59.8$ & $283.8 \pm 59.5$ & $284.5 \pm 59.8$ & 0.4 & $278.2 \pm 59.4$ & $280.1 \pm 60.3$ & $278.0 \pm 59.3$ & $<0.0001$ \\
\hline $\begin{array}{l}\text { Pre-operative } \\
\text { BMI }\end{array}$ & $46.3 \pm 8.2$ & $46.0 \pm 8.2$ & $46.4 \pm 8.2$ & 0.007 & $45.2 \pm 8.0$ & $45.5 \pm 8.2$ & $45.2 \pm 8.0$ & $<0.0001$ \\
\hline \multicolumn{9}{|c|}{ Categorical variables, $N(\%)$} \\
\hline \multicolumn{9}{|c|}{ ASA class } \\
\hline 1 & $163(0.2)$ & $30(0.5)$ & $133(0.2)$ & $<0.0001$ & $724(0.4)$ & $42(0.3)$ & $682(0.4)$ & $<0.0001$ \\
\hline 2 & $13,438(17.2)$ & $1002(17.2)$ & $12,436(17.2)$ & & $48,014(25.3)$ & $3036(23.5)$ & $44,978(25.5)$ & \\
\hline 3 & $60,927(78.1)$ & $4561(78.4)$ & $56,366(78.1)$ & & $134,533(71.0)$ & $9403(72.8)$ & $125,130(70.9)$ & \\
\hline 4 & $3458(4.4)$ & $224(3.9)$ & $3234(4.5)$ & & $6220(3.3)$ & $431(3.3)$ & $5789(3.3)$ & \\
\hline 5 & $5(0.01)$ & 0 & $5(0.01)$ & & $12(0.01)$ & - & $12(0.01)$ & \\
\hline \multicolumn{9}{|l|}{ Gender } \\
\hline Male & $15,691(20.1)$ & $1180(20.3)$ & $14,511(20.1)$ & 0.7 & $39,852(21.0)$ & $2,579(20.0)$ & $37,273(21.1)$ & 0.002 \\
\hline Female & $62,300(79.88)$ & 4637 (79.7) & $57,663(79.9)$ & 0.7 & $149,651(79.0)$ & $10,333(80.0)$ & $139,318(78.9)$ & 0.002 \\
\hline \multicolumn{9}{|l|}{ Race } \\
\hline $\begin{array}{l}\text { Non- } \\
\text { Hispanic } \\
\text { White }\end{array}$ & $52,145(66.9)$ & $4071(70.0)$ & $48,071(66.6)$ & $<0.0001$ & $120,250(63.5)$ & $8273(64.1)$ & $111,977(63.4)$ & 0.1 \\
\hline $\begin{array}{l}\text { Non- } \\
\text { Hispanic } \\
\text { Black }\end{array}$ & $10,586(13.6)$ & $891(15.3)$ & $9695(13.4)$ & $<0.0001$ & $34,856(18.4)$ & $2592(20.1)$ & $32,264(18.3)$ & $<0.0001$ \\
\hline Hispanic & $9587(12.3)$ & $571(9.8)$ & $9016(12.5)$ & $<0.0001$ & $23,340(12.3)$ & $1426(11.0)$ & $21,914(12.4)$ & $<0.0001$ \\
\hline \multicolumn{9}{|c|}{ Pre-operative comorbidities, $N(\%)$} \\
\hline $\begin{array}{l}\text { History of } \\
\text { MI }\end{array}$ & $1,284(1.7)$ & $97(1.7)$ & $1,187(1.6)$ & $<0.0001$ & $2,298(1.2)$ & $158(1.2)$ & $2,140(1.2)$ & 0.9 \\
\hline $\begin{array}{l}\text { Hyperten- } \\
\text { sion } \\
\text { requiring } \\
\text { medica- } \\
\text { tion }\end{array}$ & $41,644(53.4)$ & $3337(57.4)$ & $38,307(53.1)$ & $<0.0001$ & $89,404(47.2)$ & $6160(47.7)$ & $83,244(47.1)$ & 0.2 \\
\hline $\begin{array}{l}\text { Hyperlipi- } \\
\text { demia }\end{array}$ & $23,014(29.5)$ & $1884(32.4)$ & $21,130(29.3)$ & $<0.0001$ & $42,946(22.7)$ & 2952 (22.9) & 39,994 (22.7) & 0.6 \\
\hline $\begin{array}{l}\text { Renal insuf- } \\
\text { ficiency }\end{array}$ & $510(0.7)$ & $54(0.9)$ & $456(0.6)$ & 0.007 & $1,228(0.7)$ & $96(0.7)$ & $1,132(0.6)$ & 0.2 \\
\hline Dialysis & $128(0.2)$ & $12(0.2)$ & $116(0.2)$ & 0.4 & $592(0.3)$ & $45(0.6)$ & $547(0.3)$ & 0.4 \\
\hline $\begin{array}{l}\text { DVT } \\
\text { requiring } \\
\text { therapy }\end{array}$ & 1440 (1.9) & 91 (1.6) & 1349 (1.9) & 0.09 & 2797 (1.48) & 204 (1.6) & $2593(1.5)$ & 0.3 \\
\hline $\begin{array}{l}\text { History of } \\
\text { PE }\end{array}$ & $974(1.3)$ & $70(1.2)$ & $904(1.3)$ & 0.7 & $2,070(1.1)$ & $146(1.1)$ & 1924 (1.09) & 0.7 \\
\hline $\begin{array}{l}\text { Limited } \\
\text { ambulation } \\
\text { status }\end{array}$ & 1634 (2.1) & $185(3.2)$ & $1449(2.0)$ & $<0.0001$ & 3108 (1.6) & $344(2.7)$ & 2764 (1.6) & $<0.0001$ \\
\hline $\begin{array}{l}\text { Partial } \\
\text { functional } \\
\text { depend- } \\
\text { ence }\end{array}$ & $609(0.8)$ & $64(1.1)$ & $545(0.8)$ & 0.004 & $1206(0.6)$ & $88(0.7)$ & $1118(0.6)$ & 0.5 \\
\hline
\end{tabular}


Table 1 (continued)

\begin{tabular}{|c|c|c|c|c|c|c|c|c|}
\hline & \multicolumn{4}{|c|}{ Roux-en- Y gastric bypass } & \multicolumn{4}{|c|}{ Sleeve gastrectomy } \\
\hline & All $[\mathrm{n}=77,991]$ & RGB $[\mathrm{n}=5817]$ & $\begin{array}{l}\text { LGB } \\
{[n=72,174]}\end{array}$ & P-value & $\begin{array}{l}\text { All } \\
{[n=189,503]}\end{array}$ & $\begin{array}{l}\text { RSG } \\
{[n=12,912]}\end{array}$ & $\begin{array}{l}\text { LSG } \\
{[n=176,591]}\end{array}$ & $p$-value \\
\hline $\begin{array}{l}\text { Total } \\
\text { functional } \\
\text { depend- } \\
\text { ence }\end{array}$ & $175(0.2)$ & $15(0.3)$ & $160(0.2)$ & 0.6 & $753(0.4)$ & $33(0.3)$ & $720(0.4)$ & 0.008 \\
\hline $\begin{array}{l}\text { Diabetes } \\
\text { mellitus }\end{array}$ & $27,515(35.3)$ & $2205(37.9)$ & $25,310(35.1)$ & $<0.0001$ & $43,755(23.1)$ & $3071(23.8)$ & $40,684(23.0)$ & 0.05 \\
\hline $\begin{array}{l}\text { Chronic } \\
\text { steroid }\end{array}$ & $1122(1.4)$ & $94(1.6)$ & $1028(1.4)$ & 0.2 & $3289(1.7)$ & 209 (1.6) & $3080(1.7)$ & 0.3 \\
\hline Smoking & $6702(8.6)$ & $495(8.5)$ & $6207(8.6)$ & 0.8 & $16,947(8.9)$ & $1175(9.1)$ & $15,772(8.9)$ & 0.5 \\
\hline OSA & $33,791(43.3)$ & $2592(44.6)$ & $31,199(43.2)$ & 0.05 & $67,811(35.8)$ & $4775(37.0)$ & $63,036(35.7)$ & 0.003 \\
\hline COPD & $1608(2.1)$ & $181(3.1)$ & $1,427(2.0)$ & $<0.0001$ & $3193(1.7)$ & $212(1.6)$ & $2981(1.7)$ & 0.7 \\
\hline $\begin{array}{l}\text { Oxygen- } \\
\text { dependent }\end{array}$ & $694(0.9)$ & $51(0.9)$ & $643(0.9)$ & 0.9 & $1168(0.6)$ & $61(0.5)$ & $1107(0.6)$ & $\mathbf{0 . 0 3}$ \\
\hline
\end{tabular}

Bold values represent descriptive statistics and outcome variables that were significantly different between the study cohorts

$R G B$ robotic-assisted gastric bypass, $L G B$ conventional laparoscopic gastric bypass, $R S G$ robotic-assisted sleeve gastrectomy, $L S G$ conventional laparoscopic sleeve gastrectomy, $S D$ standard deviation, $l b s$ pounds, $B M I$ body mass index, $A S A$ American Society of Anesthesiologist, $M I$ myocardial infarction, $D V T$ deep venous thrombosis, $P E$ pulmonary emboli, $O S A$ obstructive sleeve apnea, $C O P D$ chronic obstructive pulmonary disease

bypass cases, 30 -day readmission $(7.2 \%$ vs. $5.7 \%, p=0.02)$ remained significantly higher in the robotic-assisted cohort, while aggregate SSI $(1.5 \%$ vs. $0.6 \%, p=0.003)$ remained higher in the conventional laparoscopic cohort. In this analysis, post-operative length of stay, mortality, transfusion, and all other aggregate complications remained lower in the robotic-assisted gastric bypass cohort, but the differences were not statistically significant. Renal and VTE complications remained higher in the robotic-assisted cohort, but the differences were also not statistically significant. For sleeve gastrectomy cases, post-operative length of stay and all 30-day outcomes were similar after controlling for operation length and conversion as surrogates for surgeon experience. Drain present at 30 -days $(0.6 \%$ vs. $0.1 \%, p<0.0001)$ and post-operative sepsis $(0.1 \%$ vs. $0.05 \%, p=0.01)$ remained higher in the robotic-assisted sleeve gastrectomy group. Intervention and aggregate bleeding, which were previously significantly higher in the conventional laparoscopic cohort, were now similar between these cohorts. Post-operative transfusion requirement, which was previous non-significantly higher in the laparoscopic cohort, was now significantly higher in this cohort $(0.5 \%$ vs. $0.2 \%, p=0.01)$. All other outcome measures were similar between cohorts.

\section{Discussion}

Approaches to metabolic and bariatric surgery continue to evolve. Over the last decade, robotic-assisted Roux-en$\mathrm{Y}$ gastric bypass and sleeve gastrectomy are increasingly performed with varied results [4, 9-20]. Recent meta-analyses comparing RBS and LBS have reported no significant differences between major and minor complications, 30-day reoperation, or readmission rates; however, they are limited by the small sample size and heterogeneity of the studies included in the meta-analysis $[9,10]$. In the review by Bailey et al., the authors concluded that the "review found the evidence insufficient to show a difference in complications, operative outcomes, or length of stay between roboticassisted and laparoscopic gastric bypass [9]. In contrast, $\mathrm{Li}$ et al. reported a significant difference in post-operative length of stay (HLOS) and operative time for the robotic cohort, but no statistical difference in complications or readmission rates [10]. In a recent multivariate regression analysis of the 2015 MBSAQIP PUF database comparing roboticassisted and laparoscopic sleeve gastrectomy $(n=75,079)$, Alizadeh et al. found that 30-day reoperation, intervention, and readmission rates were higher in the robotic cohort [11].

Here, we attempted to add to the published evidence by comparing metabolic and bariatric surgery outcomes performed with conventional laparoscopic and robotic-assisted approaches. Our study represents the largest 1:3 case-control matched analysis of the MBSAQIP PUF database comparing these two surgical platforms.

\section{Roux-en-Y gastric bypass: robotic-assisted versus conventional laparoscopic}

In our 1:3 case-control matched analysis of gastric bypass cases, we found that operative duration was significantly 
Table 2 Outcomes in unmatched Roux-en-Y gastric bypass and sleeve gastrectomy cohorts

\begin{tabular}{|c|c|c|c|c|c|c|c|c|}
\hline & \multicolumn{4}{|c|}{ Roux-en-Y gastric bypass } & \multicolumn{4}{|c|}{ Sleeve gastrectomy } \\
\hline & All $[n=77,991]$ & RGB $[n=5817]$ & $\begin{array}{l}\text { LGB } \\
{[n=72,174]}\end{array}$ & $p$-Value & $\begin{array}{l}\text { All } \\
{[n=189,503]}\end{array}$ & $\begin{array}{l}\text { RSG } \\
{[n=12,912]}\end{array}$ & $\begin{array}{l}\text { LSG } \\
{[n=176,591]}\end{array}$ & $p$-Value \\
\hline $\begin{array}{l}\text { Operative } \\
\text { length in } \\
\text { minutes } \\
(\text { mean } \pm \mathrm{SD})\end{array}$ & $119.6 \pm 54.1$ & $154 \pm 63.1$ & $116.8 \pm 52.3$ & $<0.0001$ & $74.3 \pm 37.2$ & $100.6 \pm 44.1$ & $72.3 \pm 36$ & $<0.0001$ \\
\hline $\begin{array}{l}\text { Length of } \\
\text { stay in days } \\
(\text { mean } \pm S D)\end{array}$ & $2.1 \pm 2.0$ & $2.1 \pm 2.0$ & $2.1 \pm 2.0$ & 1.0 & $1.6 \pm 1.5$ & $1.8 \pm 1.7$ & $1.6 \pm 1.5$ & $<0.0001$ \\
\hline $\begin{array}{l}\text { Conversion, } n \\
(\%)\end{array}$ & $212(0.3)$ & $26(0.5)$ & $186(0.3)$ & 0.008 & $179(0.1)$ & $64(0.5)$ & $115(0.1)$ & $<0.0001$ \\
\hline \multicolumn{9}{|c|}{ 30-day outcomes and peri-operative complications, N (\%) } \\
\hline $\begin{array}{l}\text { ICU admis- } \\
\text { sion }\end{array}$ & $915(1.2)$ & $65(1.1)$ & $850(1.2)$ & 0.7 & $991(0.5)$ & $73(0.6)$ & $918(0.5)$ & 0.5 \\
\hline Reoperation & $1776(2.3)$ & $149(2.6)$ & $1627(2.3)$ & 0.1 & $1628(0.9)$ & $113(0.9)$ & $1515(0.9)$ & 0.8 \\
\hline Readmission & $4766(6.1)$ & $406(7.0)$ & $4360(6.0)$ & 0.004 & $6078(3.2)$ & 460 (3.6) & $5618(3.2)$ & 0.02 \\
\hline Intervention & 2004 (2.6) & $171(2.9)$ & $1833(2.5)$ & 0.06 & $1821(1.0)$ & $164(1.3)$ & $1657(0.9)$ & 0.0002 \\
\hline Mortality & $119(0.2)$ & $6(0.1)$ & $113(0.2)$ & 0.3 & $140(0.07)$ & $8(0.06)$ & $132(0.07)$ & 0.6 \\
\hline $\begin{array}{l}\text { Related mor- } \\
\text { tality }\end{array}$ & $59(0.08)$ & $3(0.05)$ & $56(0.08)$ & 1.0 & $66(0.03)$ & $3(0.02)$ & $63(0.04)$ & 0.6 \\
\hline $\begin{array}{l}\text { Operative } \\
\text { drain present }\end{array}$ & $510(0.7)$ & $15(0.3)$ & $495(0.7)$ & $<0.0001$ & $396(0.2)$ & $63(0.5)$ & $333(0.2)$ & $<0.0001$ \\
\hline Renal failure & $103(0.1)$ & $6(0.1)$ & $97(0.1)$ & 0.5 & $108(0.06)$ & $6(0.05)$ & $102(0.06)$ & 0.6 \\
\hline $\begin{array}{l}\text { Progressive } \\
\text { renal insuf- } \\
\text { ficiency }\end{array}$ & $81(0.1)$ & $5(0.09)$ & $76(0.1)$ & 0.7 & $107(0.06)$ & $5(0.04)$ & $102(0.06)$ & 0.4 \\
\hline CPR & $45(0.06)$ & $3(0.05)$ & $42(0.06)$ & 0.8 & $59(0.03)$ & $3(0.02)$ & $56(0.03)$ & 0.6 \\
\hline Coma $>24 \mathrm{~h}$ & $1(0.001)$ & - & $1(0.00)$ & 0.8 & $7(0.004)$ & - & $7(0.004)$ & 0.5 \\
\hline Stroke/CVA & $3(0.004)$ & - & $3(0.00)$ & 0.6 & $23(0.01)$ & $2(0.02)$ & $21(0.01)$ & 0.7 \\
\hline MI & $42(0.05)$ & $2(0.03)$ & $40(0.06)$ & 0.5 & $54(0.03)$ & $4(0.03)$ & $50(0.03)$ & 0.9 \\
\hline DVT & $135(0.2)$ & $6(0.1)$ & $129(0.2)$ & 0.2 & $330(0.2)$ & $24(0.2)$ & $306(0.2)$ & 0.7 \\
\hline $\begin{array}{l}\text { Pulmonary } \\
\text { embolism }\end{array}$ & $117(0.2)$ & $8(0.1)$ & $109(0.2)$ & 0.8 & $172(0.1)$ & $13(0.1)$ & 159 (0.09) & 0.7 \\
\hline $\begin{array}{l}\text { Antico- } \\
\text { agulation for } \\
\text { DVT/PE }\end{array}$ & $341(0.4)$ & $26(0.5)$ & $315(0.4)$ & 0.9 & $823(0.4)$ & $57(0.4)$ & $766(0.4)$ & 0.9 \\
\hline Transfusion & $859(1.1)$ & $36(0.6)$ & $823(1.1)$ & 0.0002 & $931(0.5)$ & $50(0.4)$ & $881(0.5)$ & 0.08 \\
\hline $\begin{array}{l}\text { Post-operative } \\
\text { pneumonia }\end{array}$ & $327(0.4)$ & $20(0.3)$ & $307(0.4)$ & 0.4 & $256(0.1)$ & $21(0.2)$ & $235(0.1)$ & 0.4 \\
\hline $\begin{array}{l}\text { On ventila- } \\
\text { tor }>48 \mathrm{~h}\end{array}$ & $130(0.2)$ & $6(0.1)$ & $127(0.2)$ & 0.2 & $98(0.05)$ & $15(0.1)$ & $83(0.05)$ & 0.0008 \\
\hline $\begin{array}{l}\text { Unplanned } \\
\text { intubation }\end{array}$ & $213(0.3)$ & $13(0.2)$ & $200(0.3)$ & 0.5 & $223(0.1)$ & $20(0.2)$ & $203(0.1)$ & 0.2 \\
\hline $\begin{array}{l}\text { Post-operative } \\
\text { UTI }\end{array}$ & $386(0.5)$ & $22(0.4)$ & $364(0.5)$ & 0.2 & $548(0.3)$ & $36(0.3)$ & $512(0.3)$ & 0.8 \\
\hline $\begin{array}{l}\text { Post-operative } \\
\text { sepsis }\end{array}$ & $149(0.2)$ & $10(0.2)$ & $139(0.2)$ & 0.7 & $153(0.08)$ & $18(0.1)$ & $135(0.08)$ & 0.02 \\
\hline $\begin{array}{l}\text { Post-operative } \\
\text { septic shock }\end{array}$ & $92(0.1)$ & $4(0.07)$ & $88(0.1)$ & 0.3 & $59(0.03)$ & $5(0.04)$ & $54(0.03)$ & 0.6 \\
\hline $\begin{array}{l}\text { Superficial } \\
\text { SSI }\end{array}$ & $720(0.9)$ & $17(0.3)$ & 703 (1.0) & $<0.0001$ & $430(0.2)$ & $23(0.2)$ & $407(0.2)$ & 0.2 \\
\hline Deep SSI & $118(0.2)$ & $6(0.1)$ & $112(0.2)$ & 0.3 & $45(0.02)$ & $2(0.02)$ & $43(0.02)$ & 0.5 \\
\hline $\begin{array}{l}\text { Organ space } \\
\text { SSI }\end{array}$ & $266(0.3)$ & $23(0.4)$ & $243(0.3)$ & 0.5 & $302(0.2)$ & $40(0.3)$ & $262(0.2)$ & $<0.0001$ \\
\hline
\end{tabular}


Table 2 (continued)

\begin{tabular}{|c|c|c|c|c|c|c|c|c|}
\hline & \multicolumn{4}{|c|}{ Roux-en-Y gastric bypass } & \multicolumn{4}{|c|}{ Sleeve gastrectomy } \\
\hline & All $[n=77,991]$ & RGB [ $n=5817]$ & $\begin{array}{l}\text { LGB } \\
{[n=72,174]}\end{array}$ & $p$-Value & $\begin{array}{l}\text { All } \\
{[n=189,503]}\end{array}$ & $\begin{array}{l}\text { RSG } \\
{[n=12,912]}\end{array}$ & $\begin{array}{l}\text { LSG } \\
{[n=176,591]}\end{array}$ & $p$-Value \\
\hline \multicolumn{9}{|c|}{ Aggregate complications, $N(\%)$} \\
\hline Leak & $268(0.3)$ & $22(0.4)$ & $246(0.3)$ & 0.6 & $466(0.3)$ & $48(0.4)$ & $418(0.2)$ & 0.003 \\
\hline Bleeding & $676(0.9)$ & $40(0.7)$ & $636(0.9)$ & 0.1 & $507(0.3)$ & $29(0.2)$ & $478(0.3)$ & 0.3 \\
\hline $\begin{array}{l}\text { Renal compli- } \\
\text { cations }\end{array}$ & $197(0.3)$ & $14(0.2)$ & $183(0.3)$ & 0.9 & $236(0.1)$ & $11(0.09)$ & $225(0.1)$ & 0.2 \\
\hline $\begin{array}{l}\text { Venous } \\
\text { thromboem- } \\
\text { bolic events }\end{array}$ & $486(0.7)$ & $38(0.7)$ & $448(0.6)$ & 0.8 & $1135(0.6)$ & $81(0.6)$ & $1054(0.6)$ & 0.7 \\
\hline $\begin{array}{l}\text { Cardiovascu- } \\
\text { lar compli- } \\
\text { cations }\end{array}$ & $77(0.1)$ & $6(0.1)$ & $71(0.1)$ & 0.9 & $150(0.08)$ & $7(0.05)$ & $143(0.08)$ & 0.3 \\
\hline $\begin{array}{l}\text { Pulmonary } \\
\text { complica- } \\
\text { tions }\end{array}$ & $662(0.9)$ & $43(0.7)$ & $619(0.9)$ & 0.3 & $668(0.4)$ & $59(0.5)$ & $609(0.3)$ & 0.04 \\
\hline $\begin{array}{l}\text { Wound infec- } \\
\text { tion }\end{array}$ & $1226(1.6)$ & $55(1.0)$ & $1171(1.6)$ & $<0.0001$ & $868(0.5)$ & $72(0.6)$ & $796(0.5)$ & 0.08 \\
\hline
\end{tabular}

Bold values represent descriptive statistics and outcome variables that were significantly different between the study cohorts

$R G B$ robotic-assisted gastric bypass, $L G B$ laparoscopic gastric bypass, $R S G$ robotic-assisted sleeve gastrectomy, $L S G$ laparoscopic sleeve gastrectomy, $S D$ standard deviation, $I C U$ intensive care unit, $I C U$ intensive care unit, $C P R$ cardiopulmonary arrest, $C V A$ cerebrovascular accident, $M I$ myocardial infarction, $D V T$ deep venous thrombosis, $P E$ pulmonary emboli, $U T I$ urinary tract infection, SSI surgical site infection

longer with the robotic-assisted surgical approach $(p<0.0001)$, which is consistent with the findings by $\mathrm{Li}$ et al. and others $[4,10,11,13,15,16]$. We also found a significantly higher rate of 30-day readmission in the roboticassisted gastric bypass cohort $(p=0.03)$. This is consistent with a large $(n=137,455)$ retrospective propensity-matched cohort analysis of the Bariatric Outcomes Longitudinal Database (BOLD), where Celio et al. found longer operative time, 30-day reoperation, and 30-day readmission in the robotic-assisted RnYGB cohort [18]. However, after the authors controlled for operative time, 30-day readmission was no longer significant, which is inconsistent with our findings. In our study, we have found that 30-day readmission remained significantly higher in the robotic-assisted cohort, even when controlling for operative time and conversion rate as surrogates for surgeon experience $(p=0.02)$. Post-operative length of stay between the two surgical approaches has also been a point of contention in the literature. In our study, HLOS was similar between the two surgical approaches for gastric bypass before $(p=0.2)$ and after $(p=0.08)$ controlling for operative time and conversion rate. Studies have found both shorter HLOS [13, 19] and longer HLOS [16] in robotic-assisted RnYGB cohorts, while others have reported no difference [14, 15]. While our study represents a large 1:3 case-control cohort analysis comparing surgical approaches between Roux-en-Y gastric bypass ( $n=12,688)$, and will likely add value to the discussion, the reasons for differences in readmission rates between these two surgical approaches remain unclear and in need of further study.

In our case-control matched analysis of robotic and laparoscopic gastric bypass, we found significantly lower rates of mortality $(p=0.05)$, transfusion $(p=0.005)$, superficial SSI $(p=0.0003)$, aggregate bleeding $(p=0.04)$, and aggregate wound infection $(p=0.006)$ in the robotic cohort. After controlling for operative time and conversion rate, however, only superficial SSI $(p=0.0002)$ and aggregate wound infection ( $p=0.003$ ) remained lower in the robotic-assisted cohort. Regarding 30-day mortality, others have reported no significant difference between these surgical approaches [4, 9, 10, 12, 14-19], which is consistent with our study once we account for operative length and conversion rate. A lower rate of SSI following roboticassisted compared to conventional laparoscopic gastric bypass has not been previously reported [9, 10, 12-14, 16, 19]. Similar to our findings, Sebastian et al. analyzed the same data set with propensity-score matching and reported higher rates of bleeding following laparoscopic gastric bypass before and after adjusting for operative time and conversion rate [19]. However, once conversion rate and operative length is accounted for in our case-control study, we have shown no difference in transfusion rate between the two surgical approaches $(p=0.1)$. This trend toward higher transfusion rate with conventional laparoscopic compared to robotic-assisted RnYGB remains unclear. Possible mechanism may include a higher rate of stapled 
Table 3 Descriptive statistics after 1:3 case-control matching in Roux-en-Y gastric bypass and sleeve gastrectomy cohorts

\begin{tabular}{|c|c|c|c|c|c|c|}
\hline & \multicolumn{3}{|c|}{ Roux-en-Y gastric bypass $[n=12,688]$} & \multicolumn{3}{|c|}{ Sleeve gastrectomy $[n=35,956]$} \\
\hline & RGB $[n=3172]$ & LGB $[n=9516]$ & $p$-Value & RSG [ $n=8989]$ & $\operatorname{LSG}[n=26,967]$ & $p$-Value \\
\hline \multicolumn{7}{|l|}{ Continuous variables, mean $\pm \mathrm{SD}$} \\
\hline Age (years) & $44.1 \pm 11.6$ & $44.12 \pm 11.5$ & 1.0 & $43.0 \pm 11.7$ & $43.0 \pm 11.6$ & 0.7 \\
\hline Pre-operative weight & $283.3 \pm 58.7$ & $284 \pm 60.2$ & 0.6 & $278.3 \pm 58.9$ & $278.0 \pm 59.1$ & 0.7 \\
\hline Pre-operative BMI & $46.2 \pm 7.7$ & $46.49 \pm 8.0$ & 0.09 & $45.3 \pm 7.6$ & $45.4 \pm 7.6$ & 0.8 \\
\hline \multicolumn{7}{|l|}{ Categorical variables, $N(\%)$} \\
\hline \multicolumn{7}{|l|}{ ASA class } \\
\hline 1 & $1(0.03)$ & $3(0.03)$ & 1.0 & $6(0.07)$ & $18(0.07)$ & 1.0 \\
\hline 2 & $495(15.6)$ & $1485(15.6)$ & & $2210(24.6)$ & $6630(24.6)$ & \\
\hline 3 & $2641(83.3)$ & $7923(83.3)$ & & $6675(74.3)$ & $20,025(74.3)$ & \\
\hline 4 & $35(1.1)$ & $105(1.1)$ & & $98(1.1)$ & $294(1.1)$ & \\
\hline \multicolumn{7}{|l|}{ Gender } \\
\hline Male & $452(14.3)$ & $1,356(14.3)$ & 1.0 & $1458(16.2)$ & $4374(16.2)$ & 1.0 \\
\hline Female & $2720(85.8)$ & $8160(85.8)$ & & $7531(83.8)$ & $22,593(83.8)$ & \\
\hline \multicolumn{7}{|l|}{ Race } \\
\hline Non-Hispanic White & $2464(77.7)$ & $7389(77.7)$ & 1.0 & 6155 (68.5) & $18,465(68.5)$ & 1.0 \\
\hline Non-Hispanic Black & $396(12.5)$ & $1188(12.5)$ & & $1671(18.6)$ & $5013(18.6)$ & \\
\hline Hispanic & $227(7.2)$ & $681(7.2)$ & & $860(9.6)$ & $2,580(9.6)$ & \\
\hline \multicolumn{7}{|l|}{ Pre-operative comorbidities, $N(\%)$} \\
\hline History of MI & $6(0.2)$ & $18(0.2)$ & 1.0 & $11(0.1)$ & $33(0.1)$ & 1.0 \\
\hline Hypertension requiring medication & $1575(49.7)$ & 4725 (49.7) & 1.0 & $3815(42.4)$ & $11,445(42.4)$ & 1.0 \\
\hline Hyperlipidemia & $710(22.4)$ & $2130(22.4)$ & 1.0 & $1448(16.1)$ & $4344(16.1)$ & 1.0 \\
\hline Renal insufficiency & $1(0.03)$ & $3(0.03)$ & 1.0 & - & - & - \\
\hline Vein thrombosis requiring therapy & - & - & - & $5(0.06)$ & $15(0.06)$ & 1.0 \\
\hline History of PE & $1(0.03)$ & $3(0.03)$ & 1.0 & $4(0.04)$ & $12(0.04)$ & 1.0 \\
\hline Limited ambulation status & $2(0.06)$ & $6(0.06)$ & 1.0 & $12(0.1)$ & $36(0.1)$ & 1.0 \\
\hline Diabetes mellitus & $831(26.2)$ & $2493(26.2)$ & 1.0 & $1391(15.5)$ & $4173(15.5)$ & 1.0 \\
\hline Steroid/immunosuppressant use & $1(0.03)$ & $3(0.03)$ & 1.0 & $537(6.0)$ & $1,611(6.0)$ & 1.0 \\
\hline Current smoker within 1-year & $133(4.2)$ & $399(4.2)$ & 1.0 & $21(0.2)$ & $63(0.2)$ & 1.0 \\
\hline Obstructive sleep apnea & $1,239(39.1)$ & $3,717(39.1)$ & 1.0 & $2,845(31.7)$ & $8,535(31.7)$ & 1.0 \\
\hline Chronic obstructive pulmonary disease & $2(0.06)$ & $6(0.06)$ & 1.0 & $10(0.1)$ & $30(0.1)$ & 1.0 \\
\hline
\end{tabular}

$R G B$ robotic gastric bypass, $L G B$ conventional laparoscopic gastric bypass, $S D$ standard deviation, $l b s$ pounds, $B M I$ body mass index, $A S A$ American Society of Anesthesiologist, $M I$ myocardial infarction, $P E$ pulmonary emboli

anastomosis with conventional laparoscopic compared to a higher rate of hand-sewn anastomosis with the roboticassisted approach. Surgeons may also be more aggressive in suture hemostasis with the robotic-assisted approach due to easier ergonomics and improved visualization with the robotic platform. These patient level data points are not available in this database, and are worthy of future studies. However, even with a significantly higher operative duration, overall complications were less with the robotic-assisted approach for gastric bypass. In our second matched analysis, controlling for operation duration and conversion, there was much less outcome differences between robotic-assisted gastric bypass and conventional laparoscopic gastric bypass, with similar mortality and morbidity rates.

\section{Sleeve gastrectomy: robotic-assisted versus conventional laparoscopic}

In our matched analysis of over 35,000 sleeve gastrectomy cases, we found that robotic-assisted SG was associated with significantly longer operative duration $(p<0.0001)$ and post-operative length of stay $(p<0.0001)$. These findings are consistent with Sebastian et al. and their analysis of the two surgical approaches in SG, as well as a systematic review and meta-analysis [19, 20]. Most perioperative outcomes were similar between robotic-assisted and laparoscopic sleeve gastrectomy cohorts. Some outcomes were more common in the robotic-assisted group, including 30-day intervention $(p=0.01)$, drain present at 30-day $(p<0.0001)$, sepsis $(p=0.01)$ and organ space SSI 
Table 4 Outcomes after 1:3 case-control matching in Roux-en-Y gastric bypass and sleeve gastrectomy cohorts

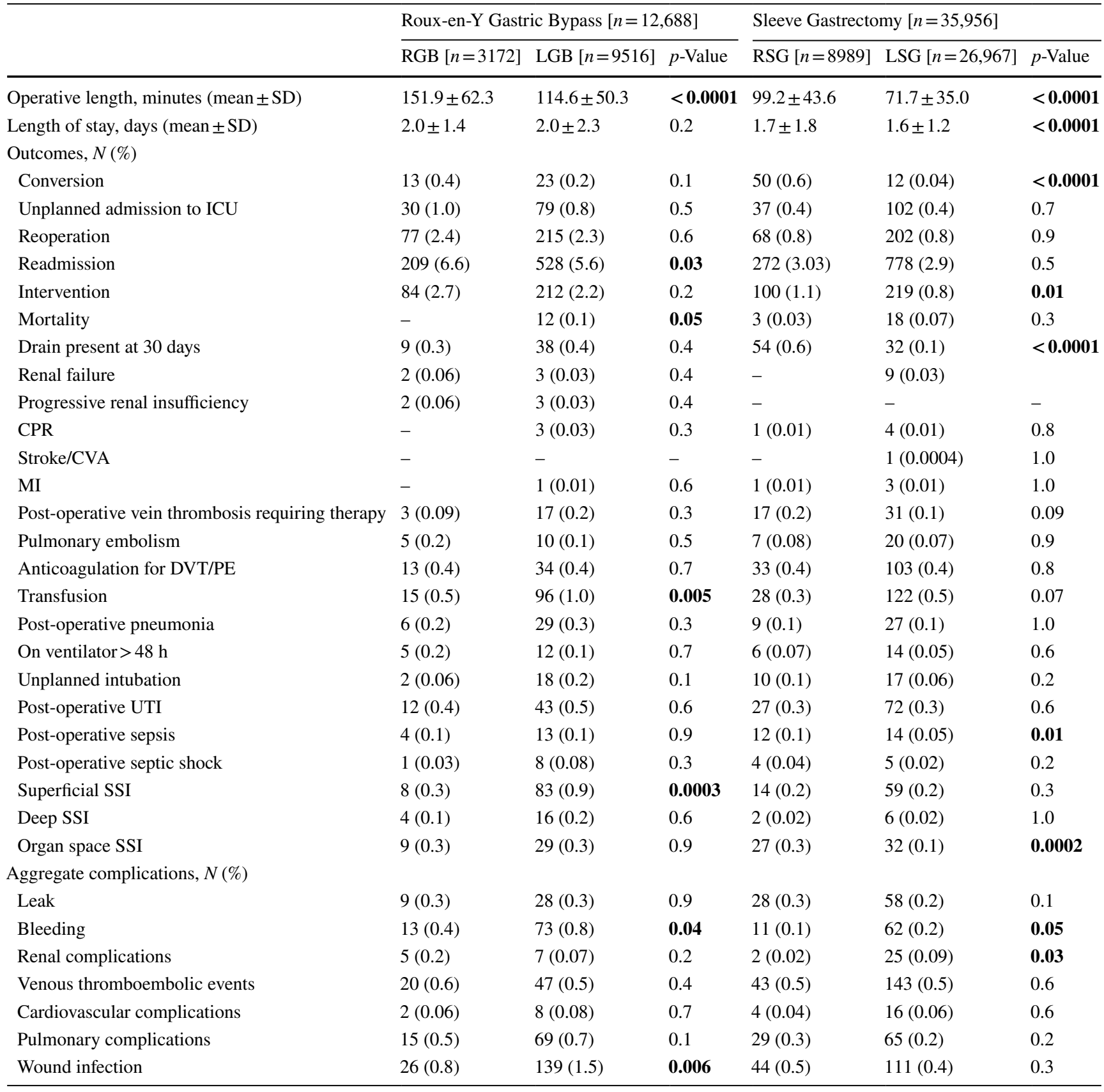

Bold values represent descriptive statistics and outcome variables that were significantly different between the study cohorts

$R G B$ robotic-assisted gastric bypass, $L G B$ conventional laparoscopic gastric bypass, $R S G$ robotic-assisted sleeve gastrectomy, $L S G$ conventional laparoscopic sleeve gastrectomy, $S D$ standard deviation, $I C U$ intensive care unit, $C P R$ cardiopulmonary arrest, $C V A$ cerebrovascular accident, $M I$ myocardial infarction, $D V T$ deep venous thrombosis, $P E$ pulmonary emboli, $U T I$ urinary tract infection, SSI surgical site infection

$(p=0.0002)$. While we did not find higher rates of readmission and reoperation in our robotic-assisted sleeve gastrectomy cohort, as did Alizadeh et al. [11], we similarly found a higher rate of organ space SSI $(p=0.0002)$. While leak rate was higher in our robotic-assisted cohort, the difference was not statistically significant $(p=0.1)$, which is similar to other published reports $[10,19,20]$, and in contrast to the significantly higher leak rate noted in the study by Alizadeh et al. [11].

Similar to analysis of our gastric bypass cases, we found a lower rate of transfusion $(p=0.07)$ and aggregate bleeding complications $(p=0.05)$ in our robotic-assisted sleeve gastrectomy cohort. Aggregate renal complications were also noted to be 4.5 -fold lower in robotic cohort of 
Table 5 Outcomes after 1:3 case-control matching in Roux-en-Y gastric bypass and sleeve gastrectomy cohorts, stratified by operative length and conversion rate

\begin{tabular}{|c|c|c|c|c|c|c|}
\hline & \multicolumn{3}{|c|}{ Roux-en-Y gastric bypass $[n=7576]$} & \multicolumn{3}{|c|}{ Sleeve gastrectomy $[n=25,164]$} \\
\hline & RGB $[n=1894]$ & LGB $[n=5682]$ & $p$-Value & $\operatorname{RSG}[n=6291]$ & LSG $[n=18,873]$ & $p$-Value \\
\hline Operative length, minutes (mean \pm SD) & $146.6 \pm 59.7$ & $139.1 \pm 49.2$ & 1.0 & $95.6 \pm 42.4$ & $90.12 \pm 38.3$ & 1.0 \\
\hline Length of Stay in days (mean \pm SD) & $2.0 \pm 1.4$ & $2.1 \pm 2.8$ & 0.08 & $1.7 \pm 1.7$ & $1.7 \pm 1.4$ & 0.6 \\
\hline \multicolumn{7}{|l|}{ Outcome, $N(\%)$} \\
\hline Unplanned ICU admission & $21(1.1)$ & $44(0.8)$ & 0.2 & $21(0.3)$ & $83(0.4)$ & 0.3 \\
\hline Reoperation & $51(2.7)$ & $113(2.0)$ & 0.07 & $49(0.8)$ & $153(0.8)$ & 0.8 \\
\hline Readmission & $137(7.2)$ & $325(5.7)$ & 0.02 & $185(2.9)$ & $569(3.01)$ & 0.8 \\
\hline Intervention & $55(2.9)$ & $149(2.6)$ & 0.5 & $66(1.1)$ & $174(0.92)$ & 0.4 \\
\hline Mortality & - & $8(0.1)$ & 0.1 & $3(0.05)$ & $9(0.05)$ & 1.0 \\
\hline Related mortality & - & $3(0.05)$ & - & $2(0.03)$ & $5(0.03)$ & 0.7 \\
\hline Drain present at 30 days & $7(0.4)$ & $22(0.4)$ & 0.9 & $40(0.6)$ & $22(0.1)$ & $<0.0001$ \\
\hline Acute renal failure & $1(0.05)$ & $2(0.04)$ & 0.7 & & $7(0.04)$ & 0.1 \\
\hline Progressive renal insufficiency & $1(0.05)$ & $2(0.04)$ & 0.7 & $1(0.02)$ & $8(0.04)$ & 0.3 \\
\hline CPR & - & $2(0.04)$ & 0.4 & - & $4(0.02)$ & 0.2 \\
\hline Stroke/CVA & - & $1(0.02)$ & 0.6 & $1(0.02)$ & - & 0.08 \\
\hline Intra-operative or post-operative MI & - & - & - & - & $2(0.01)$ & 0.4 \\
\hline Post-operative vein thrombosis requiring therapy & $1(0.05)$ & $7(0.1)$ & 0.4 & $12(0.2)$ & $37(0.2)$ & 0.9 \\
\hline Pulmonary embolism & $2(0.1)$ & $2(0.04)$ & 0.2 & $5(0.08)$ & $22(0.1)$ & 0.4 \\
\hline Anticoagulation for presumed DVT/PE & $7(0.4)$ & $13(0.2)$ & 0.2 & $24(0.4)$ & $71(0.4)$ & 1.0 \\
\hline Transfusion & $8(0.4)$ & $42(0.7)$ & 0.1 & $15(0.2)$ & $93(0.5)$ & 0.01 \\
\hline Post-operative pneumonia & $5(0.3)$ & $16(0.3)$ & 0.9 & $5(0.08)$ & $28(0.2)$ & 0.2 \\
\hline On ventilator $>48 \mathrm{~h}$ & $3(0.2)$ & $4(0.07)$ & 0.3 & $3(0.05)$ & $7(0.04)$ & 0.7 \\
\hline Unplanned intubation & $2(0.1)$ & $11(0.2)$ & 0.4 & $5(0.08)$ & $16(0.08)$ & 0.9 \\
\hline Post-operative UTI & $10(0.5)$ & $34(0.6)$ & 0.7 & $20(0.3)$ & $64(0.3)$ & 0.8 \\
\hline Post-operative sepsis & $2(0.1)$ & $10(0.2)$ & 0.5 & $10(0.1)$ & $10(0.05)$ & 0.01 \\
\hline Post-operative septic shock & - & $4(0.07)$ & 0.2 & $3(0.05)$ & $7(0.04)$ & 0.7 \\
\hline Superficial SSI & $3(0.2)$ & $60(1.1)$ & 0.0002 & $9(0.1)$ & $43(0.2)$ & 0.2 \\
\hline Deep SSI & $3(0.2)$ & $9(0.2)$ & 1.0 & $1(0.02)$ & $4(0.02)$ & 0.8 \\
\hline Organ space SSI & $3(0.2)$ & $12(0.2)$ & 0.7 & $18(0.3)$ & $31(0.2)$ & 0.06 \\
\hline Nerve injury & $1(0.05)$ & - & 0.08 & - & - & - \\
\hline \multicolumn{7}{|l|}{ Aggregate complications, $N(\%)$} \\
\hline Anastomotic leak & $5(0.3)$ & $20(0.4)$ & 0.6 & $19(0.3)$ & $38(0.2)$ & 0.1 \\
\hline Bleeding & $8(0.4)$ & $37(0.7)$ & 0.3 & $10(0.2)$ & $44(0.2)$ & 0.3 \\
\hline Renal failure & $3(0.2)$ & $4(0.07)$ & 0.3 & $1(0.02)$ & $15(0.08)$ & 0.08 \\
\hline Venous thromboembolic complications & $11(0.6)$ & $18(0.3)$ & 0.1 & $28(0.5)$ & $102(0.5)$ & 0.4 \\
\hline Cardiovascular complications & $1(0.05)$ & $4(0.07)$ & 0.8 & $1(0.02)$ & $10(0.05)$ & 0.2 \\
\hline Pulmonary complications & $10(0.5)$ & $37(0.7)$ & 0.6 & $15(0.2)$ & $55(0.3)$ & 0.5 \\
\hline Wound infection & $12(0.6)$ & $86(1.5)$ & 0.003 & $28(0.5)$ & $87(0.5)$ & 0.9 \\
\hline
\end{tabular}

Bold values represent descriptive statistics and outcome variables that were significantly different between the study cohorts

$R G B$ robotic-assisted gastric bypass, $L G B$ conventional laparoscopic gastric bypass, $R S G$ robotic-assisted sleeve gastrectomy, $L S G$ conventional laparoscopic sleeve gastrectomy, $S D$ standard deviation, $I C U$ intensive care unit, $C P R$ cardiopulmonary arrest, $C V A$ cerebrovascular accident, $M I$ myocardial infarction, $D V T$ deep venous thrombosis, $P E$ pulmonary emboli, UTI urinary tract infection, SSI surgical site infection

patients. The reported higher rates of bleeding complications with laparoscopic compared to robotic-assisted sleeve gastrectomy remain elusive and require further study. After controlling for operative length and conversion rate in our subsequent $1: 3$ case-control matched analysis, most outcomes did not change. However, the rate of intra-operative or post-operative transfusion became significantly higher $(p=0.01)$ in the conventional laparoscopic sleeve gastrectomy cohort, which is different from findings in previous studies $[10,11,19,20]$. 
Readmissions, re-interventions, operative duration and post-operative HLOS directly contribute to healthcare costs [22]. Many of the studies comparing the cost of conventional laparoscopic versus robotic-assisted surgery do not account for the large initial investment, consumables per procedures, annual maintenance, and other reusable equipment that comprise a substantial portion of the total operating costs of robotic platforms [9, 10, 23]. Because of the growing emphasis on healthcare costs, it is imperative that bariatric surgeons continue to find new and innovative ways to reduce costs associated with the utilization of differently surgical platforms for bariatric surgery without effecting patient outcomes. From our matched analysis, it would appear that even though robotic-assisted RnYGB takes longer, it is associated with better outcomes when compared to the conventional laparoscopic approach, including a lower overall mortality rate, less bleeding complications, and similar leak rates. In contrast, robotic-assisted sleeve gastrectomy is associated with a higher rate of infectious complications (sepsis and organ space SSI). For both gastric bypass and sleeve gastrectomy, the robotic platform seems to be protective of aggregate bleeding complications and transfusion requirements, but more so for gastric bypass cases. These findings remained, though less significant, after controlling for operation length and conversion as surrogate variables for surgeon experience. This suggest that the potential benefits of robotic-assisted metabolic and bariatric surgery noted in this study most likely represents an interplay between the surgical platform and surgeon experience.

There are several limitations to our study. First, this study was limited to peri-operative outcomes data only. Stratified outcomes on weight loss, long-term complications, and surgical impact on comorbid conditions could not be assessed. Second, this dataset does not provide relevant surgeon and surgical technique variables that may impact peri-operative outcomes. Surgeon experience or case volume has been shown to have a considerable effect on outcomes [14, 16] and is a variable that is missing from the data set. We attempted to control for this potential confounder by including operative length and conversion rate as matching covariates. There are limitations to using operation length and conversion as surrogate variables for surgeon experience, as there are other patient variables and intra-operative variables that may impact operative length and the likelihood of conversion. A more accurate representation of surgeon experience is needed in the MBSAQIP database to account for this possible confounder. The data also lack information on surgical technique variables between the two cohorts that could have also impacted outcomes. The portion of anastomosis created with a purely hand-sewn, stapled, or a combination of techniques is unknown. With the introduction of the robotic stapler, it is unclear how surgeon's anastomotic technique on the robotic platform may have changed and impacted outcomes. It is possible that higher rates of organ space SSI and post-operative sepsis seen in SG using the robotic-assisted surgical approach represent surgeon's early learning curve or changes in technique as technology evolves. There is likely significant intra-operative variation in technique between the two surgical approaches that is not accounted for in the database, and limits a true assessment on outcomes between laparoscopic and robotic-assisted Roux-en-Y gastric bypass and sleeve gastrectomy. As robotic platforms become more common, it is important to continue to critically assess these surgeon and surgical technique variables and their impact on outcomes. Lastly, even though this is the largest bariatric clinical database available, this is a retrospective analysis and is therefore vulnerable to biases associated with retrospective analysis of clinical databases. While we had a large volume of clinical data to analyze, there were missing data points. Data entry is limited by the timeliness and completeness of data entry by bariatric Clinical Nurse Reviewers. Given the missing data points, this is currently not a $100 \%$ capture of relevant clinical data and limits any analysis utilizing this database.

This study represents the largest case-control matched analyses of the MBSAQIP PUF database comparing these two surgical approaches for gastric bypass and sleeve gastrectomy. Both platforms are overall very safe. The roboticassisted platform is associated with longer operative duration. In spite of the longer operative time, we conclude that the robotic-assisted approach is safe and may provide some advantage for primary Roux-en-Y gastric bypass, as it is associated with less complication compared to the conventional laparoscopic approach. In contrast, the benefits of robotic-assisted sleeve gastrectomy remain unclear. Our analysis suggest that it is likely less cost-effective due to longer operative duration, post-operative length stay, and 30-days intervention. It may also be less safe due to higher rates of post-operative sepsis and drains present at 30-days, and a tendency toward a higher leak rate. The robotic platform seems to be protective of bleeding complications for both gastric bypass and sleeve gastrectomy, but may mitigate post-operative bleeding complications more in gastric bypass compared to sleeve gastrectomy cases and subsequently impact peri-operative mortality and morbidity. Further studies are warranted to better understand the costeffectiveness and safety of these surgical platforms, while addressing some of the limitations of this dataset. Surgeons need to remain safety- and cost-conscious when deciding which platform to use in performing primary metabolic and bariatric surgery.

Funding Our research was supported by the Department of Surgery at Temple University Hospital and the Lewis Katz School of Medicine at Temple University 


\section{Compliance with ethical standards}

Disclosures Drs. Edwin Acevedo, Jr., Michael Mazzei, Huaqing Zhao, Rohit Soans, Michael A. Edwards, and Mr. Xioaoning Lu have no conflicts of interest or financial ties to disclose.

\section{References}

1. World Health Organization (2018) Obesity and overweight. World Health Organization. http://www.who.int/en/news-room/factsheets/detail/obesity-and-overweight. Accessed Sept 242018

2. ASMBS. (2018). The Impact of Obesity on Your Body and Health. https://asmbs.org/patients/impact-of-obesity. Accessed Sept 242018

3. Mason EE, Ito C (1996) Gastric Bypass in Obesity. Obes Res 4(3):316-319

4. Elias AA et al (2018) Robotic-assisted bariatric surgery: case series analysis and comparison with the laparoscopic approach. Rev Col Bras Cir 45(3):e1806

5. Zhang Y, Zhu C, Wen X, Li L, Rampersad S, Lu L, Zhou D, Qian C, Cui R, Zhang M, Yang P, Qu S, Bu L (2017) Laparoscopic sleeve gastrectomy improves body composition and alleviates insulin resistance in obesity related acanthosis nigricans. Lipids Health Dis 16(1):209

6. Yska JP et al (2015) Remission of type 2 diabetes mellitus in patients after different types of bariatric surgery: a population-based cohort study in the United Kingdom. JAMA Surg 150(12):1126-1133

7. Ribaric G, Buchwald JM, McGlennon TW (2014) Diabetes and weight in comparative studies of bariatric surgery vs conventional medical therapy: a systematic review and meta-analysis. Obes Surg 24(3):437-455

8. Courcoulas AP et al (2014) Long-term outcomes of bariatric surgery: a National Institutes of Health Symposium. JAMA Surg 149(12):1323-1329

9. Bailey JG et al (2014) Robotic versus laparoscopic Roux-en-Y gastric bypass in obese adults ages 18 to 65 years: a systematic review and economic analysis. Surg Endosc 28:414-426

10. Li K et al (2016) Robotic versus laparoscopic bariatric surgery: a systematic review and meta-analysis. Obes Surg 26:3031-3044

11. Alizadeh RF et al (2018) Robotic versus laparoscopic sleeve gastrectomy: a MBSAQIP analysis. Surgical Endoscopy. 33:917-922

12. Buchs NC, Morel P, Azagury DE et al (2014) Laparoscopic versus robotic Roux-en-Y gastric bypass: lessons and long-term followup learned from a large prospective monocentric study. Obes Surg 4(12):2031-2039
13. Rogula et al (2018) Does robotic Roux-en-Y gastric bypass provide outcome advantages over standard laparoscopic approaches? Obes Surg 28(9):2589-2596

14. Smeenk RM, Van't Hof G, Elsten E, Feskens PGBM (2016) The results of 100 robotic versus 100 laparoscopic gastric bypass procedures: a single high volume centre experience. Obes Surg 26:1266-1273

15. Ahmad A, Carleton JD, Ahmad ZF, Agarwala A (2016) Laparoscopic versus robotic-assisted Roux-en-Y gastric bypass: a retrospective, single-center study of early perioperative outcomes at a community hospital. Surg Endosc 30:3792-3796

16. Wood $\mathrm{MH}$ et al (2014) A comparison of outcomes between the traditional laparoscopic and totally robotic Roux-en-Y gastric bypass procedures. J Robotic Surg 8:29-34

17. Moon RC et al (2016) Robotic Roux-en-Y Gastric Bypass, is it safer than laparoscopic bypass? Obes Surg 26:1016-1020

18. Celio AC et al (2017) Perioperative safety of laparoscopic versus robotic gastric bypass: a propensity matched analysis of early experience. Surg Obes Relat Dis. 13(11):1847-1852

19. Sebastian R, Howell MH, Chang KH, Adrales G, Magnuson T, Schweitzer M, Nguyen H (2018) Robot-assisted versus laparoscopic Roux-en-Y gastric bypass and sleeve gastrectomy: a propensity score-matched comparative analysis using the $2015=2016$ MBSAQIP database. Surg Endosc 33(5):1600-1612. https://doi. org/10.1007/s00464-018-6422-7

20. Magouliotis DE, Tasiopoulou VS, Sioka E, Zacharoulis D (2017) Robotic versus laparoscopic sleeve gastrectomy for morbid obesity: a systematic review and meta-analysis. Obes Surg 17(1):245-253

21. Berger ER et al (2016) The Impact of Different Surgical Techniques on Outcomes in Laparoscopic Sleeve Gastrectomies: The First Report from the Metabolic and Bariatric Surgery Accreditation and Quality Improvement Program (MBSAQIP). Ann Surg 264(3):464-473

22. Higgins RM, Frelich MJ, Bosler ME, Gould JC (2017) Cost analysis of robotic versus laparoscopic general surgery procedures. Surg Endosc 13(1):185-192

23. Ho C, Tsakonas E, Tran K, et al (2017) Robot-assisted surgery compared with open surgery and laparoscopic surgery: clinical effectiveness and economic analyses. Canadian Agency for Drugs and Technologies in Health; (CADTH Technology Report, No. 137). Table 16, Capital and Operating Costs of da Vinci Surgical System

Publisher's Note Springer Nature remains neutral with regard to jurisdictional claims in published maps and institutional affiliations. 